\title{
1 A spark of 3D revisualization: new method for re-exploring
}

\section{2 segmented data}

4 Yuzhi Hu${ }^{1}$, A. Limaye ${ }^{2}$, Jing $\mathrm{Lu}^{3,4 *}$

$5 \quad{ }^{1}$ Department of Applied Mathematics, Research School of Physics, Australian National

6 University, Canberra, ACT, Australia 2601

$7 \quad{ }^{2}$ National Computational Infrastructure, Building 143, Corner of Ward Road and Garran

8 Road, Ward Rd, Canberra, ACT, Australia 2601

$9 \quad{ }^{3}$ Key Laboratory of Vertebrate Evolution \& Human Origins of China, Institute of Vertebrate

10 Paleontology \& Paleoanthropology, Chinese Academy of Sciences,Beijing, 100044, China

$11{ }^{4}$ CAS Center for Excellence in Life \& Paleoenvironment, Chinese Academy of Science,

12 Beijing 100044, China

*Corresponding author: lujing@ivpp.ac.cn

\section{Abstract}

17 3D scientific visualization is a popular non-destructive investigation tool, however current

18 imaging processing and $3 \mathrm{D}$ visualization software has compatibility barriers which make

19 replicability and reproducibility in research difficult. To solve this, we developed a new

20 revisualization method and demonstrated four case studies using three mainstream image

21 processing and 3D visualization software. Our method offers interchangeability amongst current image processing and 3D visualization software. 
Nowadays, 3D scientific visualization, becomes a common solution for many different purposes ${ }^{1-4}$ and one of the most useful tools for research investigations, revealing hidden information behind the data ${ }^{5-8}$. For the last two decades, researchers in the 3D scientific visualization field have been experimenting with new ways to more accurately visualize medical and scientific data-sets ${ }^{3,9-17}$. In the current workflow of 3D scientific visualization process, 3D rendered results can be generated using combinations of image processing, segmentation, and rendering techniques ${ }^{18-20}$. Current image processing and 3D visualization software lack interchangeability and contain natural barriers due to compatibility (e.g. format protection between different software), accessibility (e.g. different researchers use different software so manageability of data sharing between collaborated parties is limited) and affordability (e.g. students may not afford the license for commercial software). The vast amount of time taken to master each software and the complexity behind different algorithms or approaches, are also a concern. These barriers weaken the achievability of the transparency, replicability and reproducibility of scientific research; damage the validation of communications between collaboration of institutions and restrict the authenticity assessment of a research finding, a proposed solution or a hypothesis.

It is a trend that more publishers and journals start requesting authors to upload sufficient data to support their discoveries and ensure published findings are authentic ${ }^{14,21-24}$. A common solution is uploading surface mesh data (i.e. polygon mesh, for example Stereolithography Format (. stl) or Polygon File Format (.ply)) of the segmented results of the research object. However, this approach still restricts the capability of re-investigation by peers as surface mesh data only contains external information. A more efficient and resourcefriendly way is to use the segmented volume data, which contains both internal and external information together with the original volumetric data, however, as mentioned above, due to current restrictions and barriers, this is not currently feasible. 
Most mainstream image processing and 3D visualization software (e.g. Mimics,

50 Avizo, VG Studio) can input and output Digital Imaging and Communications in Medicine

51 (DICOM). DICOM is well known in medicine for being exchangeable between any two

52 entities in biomedical imaging and analysis software ${ }^{25}$. In medicine, segmented DICOM data

53 from a scan (e.g. ultrasound or MRI) can be used to re-analysis segmented regions of interest

54 using other software for diagnostics ${ }^{26,27}$. Thus, we developed volume exploration and

55 presentation software Drishti to solve the current barriers in image processing and 3D

56 visualization software, by implementing an ability to include both single and multiple

57 DICOM directories of segmented volumetric data from other 3D visualization software. To

58 the best of our knowledge, this method conquers current compatibility and interchangeability

59 constraints among 3D visualization software.

By testing this new revisualization method, we use four segmented volumetric data from three mainstream 3D visualization software - Mimics, VG Studio and Avizo. The four segmented volume data have been saved as single (VG Studio) and multiple DICOM (Mimics and Avizo) directories, and then imported into Drishti to transform and converted into processed volume formats which Drishti can read and process. After proceeded to the processed volume format (i.e. .pvl.nc), segmented volume data can be explored and rendered in Drishti (Fig.1 and SI Figs.2-4; see SI Fig.1 for detailed work flow). Segmented structures were assigned individual colours to differentiate them (as demonstrated in Fig.1b,d,f, and g). In Drishti, we use both 1D and 2D transfer functions with combinations of different

69 illumination methods such as applying different opacity and light volumes per layer or 70 segmented structures to revisualize the segmented volume data. Our method also allows each 71 segmented layer of the original segmentation to be shown separately in the 2D transfer 72 function window with its own voxel information (Fig.1g-h). The 2D transfer function then maps the voxel information of each layer to optical properties before applying different

74 illumination methods. Voxel intensity per layer can be read separately or together using the transfer function editor. 
Revisualized segmented volume data can be exported in three standard formats using

77 Drishti Import: RAW, grayscale 8-bits unsigned image stacks and ITK MetaImage format,

78 which are considered as standard formats that all image processing and 3D visualization

79 software can read and process. Revisualized segmented volume can also be merged with

80 original volume data for enhancement of a region-of-interest or segmented area which can

81 validate the precision and accuracy of segmented results. To achieve this, two volumes, the

82 revisualized segmented volume and the original tomogram need to be loaded together in

83 Drishti Render, rendered then saved as an Extensible Markup Language data file (i.e. .xml).

84 As demonstrated, the interchangeability between different image processing and 3D

85 visualization software is achievable with this new revisualization method. In general, this

86 method works for any software that allows DICOM as an output format. During the

87 revisualization process, 2D and 3D images, movies and other visuals can also be generated.

88 All revisualized volume data can be imported directly to Drishti Prayog and Drishti VR for

89 instant interactive display.

Our revisualization method illuminates detailed information of segmented volume

91 data through different combination of illumination algorithms and features. It utilizes

92 mainstream image processing and 3D visualization software; maximizes the 3D visualized

93 outcome of any segmented volume data while maintaining the uniformity and the

94 interoperability between different software platforms and volumetric data formats. In

95 addition, this method is the simplest way to break current restrictions and barriers due to

96 compatibility, accessibility and affordability issues while maintaining the capability of

97 producing more intuitive visual representations ensuring a more efficient collaboration

98 between parties. By introducing this new revisualization method to the community will make

99 examining current hypotheses or re-investigating published research findings are more

100 achievable and feasible. This revisualization method is simple enough to be generally applied

101 to any fields. 


\section{Material and methods}

105 Segmented volume data for revisualization Segmented volume data of the earliest

106 tetrapodomorph, Tungsenia paradoxa (IVPP V10687). Original segmentation was done in

107 Mimics $^{28}$. Segmented braincase of Erofoichthys (IVPP OV2715) was scanned at IVPP. Both

108 data were output as multiple zipped DICOM files from Mimics 18.0 (option: Export-Masks).

109 Segmented volume data of a Miocene bird Linxiavis ${ }^{29}$. Original segmentation was

110 done in Avizo. This data was output as multiple DICOM directories from Avizo 9.0 (option:

111 Export-DICOM).

112 Segmented volume data of lizard Varanus indicus (AMNH R58389) was done in VG

113 Studio. This data was output as single DICOM directories from VG Studio 3.3 (option: Filter-

114 Export- volume). The CT scanning of Varanus indicus was completed at the Microscopy and

115 Imaging Facilities at the American Museum of Natural History (AMNH), with permissions

116 from the Herptetology Department of the AMNH.

The above four experiments were then imported into Drishti Import for processing.

119 Procession of segmented volume data We have implemented a new ability to import

120 multiple DICOM volume datasets and collapsing them into a single volume dataset. This was

121 done in order to accommodate the segmentation results in the form of multiple DICOM

122 volumes, each volume consisting of a single segmented structure. Each segmented structure

123 is assigned a voxel value, thereby merging all the segmented datasets into a single volumetric

124 dataset to be visualized.

We use two illumination methods in our revisualization experiments, the global

126 parameters, which include the properties of the overall scene (e.g., lights, shading, camera

127 position, projection type), and object parameters, which include the properties of the 
128 segmented object (e.g., colour and opacity). Global parameters were used to operate the scene 129 of a 3D visualization. Different mixture of parameters were used in revisualized segmented 130 volume data (see SI Figs. 2-4).

131 Image analysis Images of the revisualized volume data were generated in same orientation 132 and scale in Drishti as in Mimics, Avizo and VG Studio. Image were analysed using ImageJ 133 (https://imagej.nih.gov/ij/index.html) and ICY (http://icy.bioimageanalysis.org). Results were 134 analysed use Microsoft Excel and displayed in the method and supplementary information. 135 ImageJ (https://imagej.nih.gov/ij/index.html) and ICY (http://icy.bioimageanalysis.org/) were 136 used to analysis images to demonstrate their properties (Fig. 2 and SI Figs. 2-4). Images were 137 input into both ImageJ and ICY then transformed from RGB to 8-bits greyscale images for 138 analysis. 2D histogram of pixel intensity, 3D surface plot and edges on those images were 139 generated per image. The 2D histogram shows the distribution of grey values in that image.

140 The $\mathrm{x}$-axis represents the possible grey values and the $y$-axis shows the number of pixels 141 found for each grey value. The 3D surface plot displays a three-dimensional graph of the 142 intensities of pixels in a 8-bits greyscale colour image. Edges were calculated using Sobel 143 Edge Detection algorithm ${ }^{30}$. Results of these analysis are displayed here (Fig.2 and SI Figs.2144 4). The median optical density (OD) of each selected image (see SI Fig. 5) were 146 measured in ImageJ for further analysis and displayed in SI Fig. 5. Calibration of optical 147 density (SI Fig.8) was done using a Kodak No. 3 Calibrated Step Tablet scanned with an 148 Epson Expression 1680 Professional scanner. The tablet has 21 steps with a density range of 1490.05 to 3.05 OD (SI Fig. 8a). A weak correlation and positive linear relationship between 150 images of the revisualized segmented volume using Drishti and images of the segmented 151 volume in Mimics (SI Fig. 7). 
154 Step-table and calibrated curve for optical density and one movie for demonstration of the

155 suggested workflow of revisualization are available through Figshare:

156 https://figshare.com/s/350c258bc3d6b41313a1.

157

158 Code Availability

159 Code is available at: https://github.com/nci/Drishti

160

\section{Acknowledgements}

162 We thank Z.L. and H.Y. for sharing their segmented volume data, AMNH with permissions

163 for using their data, T.S., M.Z. and G.C.Y. for their continuous support. This research was

164 funded by the Strategic Priority Research Program of the Chinese Academy of Sciences

165 (Grant No. XDB26000000) and the National Natural Science Foundation of China

166 (41872023). Y.H. was supported by Postgraduate Research Scholarship at the Research

167 School of Physics, Australian National University.

168

169

\section{Author Contributions}

170 J.L. conceived the project. Y.H., A. J. and J.L. conducted functionality, unit and performance

171 testing for Drishti. A.L. J.L. and Y.H. implemented the revisualization process and its

172 corresponding illumination methods for DICOM directories. Y.H. and J.L. conducted image

173 analysis and interpreted the results. All authors wrote the manuscript and contributed equally

174 to this work. 
177 The authors declare no competing interests.

\section{Supplementary information}

180 Supplementary information is available to download here.

\section{References}

1831 O'Rourke, J. C., Smyth, L., Webb, A. L. \& Valter, K. How Can We Show You, If

184 You Can't See It? Trialing the Use of an Interactive Three - Dimensional Micro - CT

185 Model in Medical Education. Anat. Sci. Educ. 13, 206-217, doi:10.1002/ase.1890

$186 \quad$ (2020).

1872 Tesařová, M. et al. An interactive and intuitive visualisation method for X-ray

188 computed tomography data of biological samples in 3D Portable Document Format.

189 Sci. Rep. 9, 1-8, doi:http://dx.doi.org/10.1038/s41598-019-51180-2 (2019).

1903 Leggio, B. et al. MorphoNet: an interactive online morphological browser to explore

191 complex multi-scale data. Nat. Commun. 10, 2812, doi:10.1038/s41467-019-10668-1

192 (2019).

1934 Brodlie, K. W. et al. Scientific visualization: techniques and applications. (Springer

194 Science \& Business Media, 2012).

1955 Geier, B. et al. Spatial metabolomics of in situ host-microbe interactions at the

196 micrometre scale. Nat. Microbiol. 5, 498-510, doi:10.1038/s41564-019-0664-6

197 (2020).

1986 Johnson, E. H. \& Carter, A. M. Defossilization: A Review of 3D Printing in

199 Experimental Paleontology. Front. Ecol. Evol. 7, doi:10.3389/fevo.2019.00430

200 (2019). 
2017 Zhu, M. et al. A Silurian maxillate placoderm illuminates jaw evolution. Science 354, 202 334-336, doi:10.1126/science.aah3764 (2016).

2038 Zhu, M. et al. A Silurian placoderm with osteichthyan-like marginal jaw bones.

$204 \quad$ Nature 502, 188-193, doi:10.1038/nature12617 (2013).

2059 Johnson, C. Top scientific visualization research problems. IEEE Comput. Graph. 24, $206 \quad 13-17$ (2004).

$20710 \quad$ Hu, Y., Limaye, A. \& Lu, J. A new tool for 3D segmentation of computed 208 tomography data: Drishti Paint and its applications. Preprint at bioRxiv, 209 https://www.biorxiv.org/content/10.1101/2020.04.09.997965v2 (2020).

21011 Limaye A. Drishti: a volume exploration and presentation tool. In: Developments in 211 X-ray Tomography VIII 2012 Oct 17 (Vol. 8506, p. 85060X). SPIE, doi:

$212 \quad 10.1117 / 12.935640$. short?SSO=1 (2012).

21312 Manssour, I. H., Furuie, S. S., Olabarriaga, S. D. \& Freitas, C. M. D. S. in

214 Proceedings. XV Brazilian Symposium on Computer Graphics and Image Processing. 215 51-58 (IEEE).

21613 Miyawaki, T. et al. Visualization and molecular characterization of whole-brain 217 vascular networks with capillary resolution. Nat. Commun. 11, 1104, 218 doi:10.1038/s41467-020-14786-z (2020).

21914 Peng, H. et al. Virtual finger boosts three-dimensional imaging and microsurgery as 220 well as terabyte volume image visualization and analysis. Nat. Commun. 5, 4342, 221 doi:10.1038/ncomms5342 (2014).

22215 Rueden, C. T. et al. ImageJ2: ImageJ for the next generation of scientific image data. 223 BMC Bioinformatics 18, 529-526, doi:10.1186/s12859-017-1934-z (2017).

22416 Upson, C. et al. The application visualization system: A computational environment 225 for scientific visualization. IEEE Comput. Graph. 9, 30-42 (1989). 
22617 Yuan, J. et al. Tridimensional visualization reveals direct communication between the 227 embryo and glands critical for implantation. Nat. Commun. 9, 603, 228 doi:10.1038/s41467-018-03092-4 (2018).

22918 Lu, J. et al. A Devonian predatory fish provides insights into the early evolution of 230 modern sarcopterygians. Sci. Adv. 2, e1600154, doi:10.1126/sciadv.1600154(2016).

23119 Hu, Y., Lu, J. \& Young, G. C. New findings in a 400 million-year-old Devonian 232 placoderm shed light on jaw structure and function in basal gnathostomes. Sci. Rep. 233 7, doi:10.1038/s41598-017-07674-y (2017).

23420 Lu, J., Giles, S., Friedman, M. \& Zhu, M. A new stem sarcopterygian illuminates 235 patterns of character evolution in early bony fishes. Nat. Commun. 8, 1932-1938, 236 doi:10.1038/s41467-017-01801-z (2017).

23721 Costello, M. J. Motivating Online Publication of Data. BioScience 59, 418-427, 238 doi:10.1525/bio.2009.59.5.9 (2009).

23922 Penev, L. et al. Strategies and guidelines for scholarly publishing of biodiversity data. $240 \quad$ RIO 3, e12431 (2017).

24123 Federer, L. M. et al. Data sharing in PLOS ONE: An analysis of Data Availability 242 Statements. PLoS One 13, e0194768, doi:10.1371/journal.pone.0194768 (2018). 24324 Miyakawa, T. No raw data, no science: another possible source of the reproducibility 244 crisis. Mol. Brain 13, 24, doi:10.1186/s13041-020-0552-2 (2020).

24525 Peck, D. Digital Imaging and Communications in Medicine(DICOM): A Practical 246 Introduction and Survival Guide. J. Nucl. Med.50, 1384(2009).

24726 Shiroma, J. T. An introduction to DICOM. Vet. Med., 19-20 (2006).

248 Burgess, J. Digital DICOM in Dentistry. Open Dent. J. 9, 330-336, 249 doi:10.2174/1874210601509010330 (2015).

25028 Lu, J. et al. The earliest known stem-tetrapod from the Lower Devonian of China. 251 Nat. Commun. 3, 1160, doi:10.1038/ncomms2170(2012). 
29 Li, Z.-H., Stidham, T.A., Deng,T., Zhou, Z.H. Evidence of Late Miocene PeriTibetan Aridification From the Oldest Asian Species of Sandgrouse (Aves: Pteroclidae). Front. Ecol. Evol. 8, 59, doi: 10.3389/fevo.2020.00059(2020) (2013).

\section{Figure legends}

Figure 1. Segmented volume data from Mimics, Avizo and VG Studio and their the braincase of living fish Erofoichthys IVPP OV2715 (a, Image obtained from Mimics for segmented volume; b, image obtained from Drishti after revisualising of segmented volume). assigning individual colours to differentiate segmented structures, allowing each segmented layer of the original segmentation to be shown separately in the 2D transfer function window with its own voxel information. Three pairs of coloured arrows indicate three separate

271 revisualized segmented structures (g) and their corresponded counterparts in the 2D transfer 272 function window (h). 
278 Drishti in the same orientation and scale of a. g, Revisualized result corresponds to $\mathbf{b}$. $\mathbf{h}$,

279 Revisualized result corresponds to c. i, 2D histogram of $\mathbf{g}$. j, 3D surface plot of g. Median

280 Optical Density (OD) of $\mathbf{b}$ and $\mathbf{g}$ is 0.549 and 0.926 respectively, calculation carried

281 out using the calibrated curve displayed in SI Fig. 8b. 


\section{a}

segmented volume data

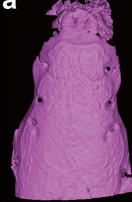

C

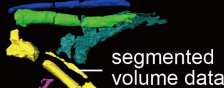

volume data

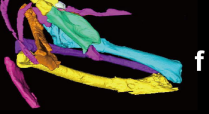

b

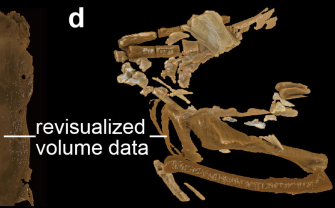

e

segmented volume data
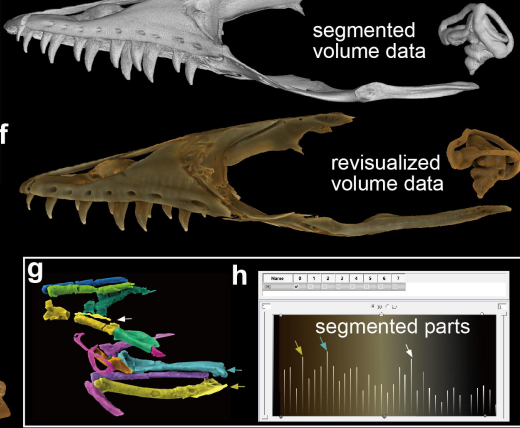


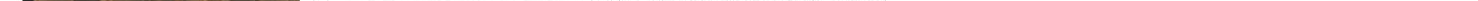

